\title{
Electron-Transfer Rate in Potential-Modulated Redox Reactions with Electro-Active Optical Waveguides
}

\author{
Xue Han and Sergio B. Mendes ${ }^{\dagger}$ \\ Department of Physics and Astronomy, University of Louisville, Louisville, KY, 40292, USA
}

\begin{abstract}
A novel methodology has been developed to determine electron-transfer rate in electrically driven redox reactions. Based on a widely adopted electrical circuit describing faradaic processes in an electrochemical cell, the approach uses a combination of impedance data from optical and electrical measurements that are simultaneously acquired in a spectroelectrochemical experiment. Once the consistency of our methodology was experimentally corroborated, it was put to practice for investigating electron-transfer rate of cytochrome $c$ adsorbates at very low concentrations on an indium tin oxide electrode by using a highly sensitive, single-mode, electro-active, integrated optical waveguide platform. Different surface densities of redox species on the electrode interface and different ionic strengths in the electrolyte solution were studied. Higher surface densities and higher ionic strengths are shown to slow down the electron-transfer process between the redox molecules and the working electrode.
\end{abstract}

Keywords Spectroelectrochemistry, electron-transfer rate, impedance spectroscopy, redox reaction, integrated optical waveguide, cytochrome $c$ protein

(Received December 31, 2016; Accepted March 10, 2017; Published April 10, 2017)

\section{Introduction}

Information on time characteristics of charge transfer events in electrically controlled redox processes is critical to identify and elucidate mechanisms underlying a particular electrochemical reaction. By modulating an electric potential applied to a working electrode, redox molecules close to the electrode surface can usually be driven back and forth through a particular oxidation state. Impedance measurements where data is collected over a range of modulation frequencies have proven useful for determination of time constants present in electrochemical processes. Usually electron-transfer kinetics of redox species needs be investigated under a variety of interfacial conditions $^{1-3}$ and electrochemical impedance spectroscopy have been widely deployed for those tasks. ${ }^{4-7}$ However, electrochemical techniques based purely on electrical measurements invariably exhibit inadequate sensitivity to detect small faradaic currents from low concentrations of redox species on the electrode surface. ${ }^{8-10}$ Non-faradaic currents from ions dissolved in the electrolyte solution typically create a strong background current (i.e., the capacitive electric double-layer current) that largely dominates the measured electrical signal. Spectroelectrochemical techniques are particularly useful to mitigate those difficulties by focusing solely on the faradaic process and avoiding the strong non-faradaic electrical background. ${ }^{10-22}$ Based on spectroscopic optical changes that typically accompany a redox event, it is usually possible to select the wavelength of a probing

† To whom correspondence should be addressed.

E-mail: sbmend01@louisville.edu

X. H. present address: Light-Matter Interaction Unit, Okinawa Institute of Science and Technology, Japan. light beam to uniquely capture the optical changes associated with the faradaic process and is immune to the presence and motion of background ions in the electric double layer.

In previous works ${ }^{23-25}$ we have described the overall design, fabrication, and application of a single-mode, electro-active, integrated optical waveguide (SM-EA-IOW) as a platform (see Fig. 1) for spectroelectrochemical investigations using optical impedance measurements of a particular type of redox event at a certain specific condition on the electrode interface. Compared to traditional single-pass transmission or single-bounce reflection techniques, the detection sensitivity of the SM-EAIOW methodology was experimentally reported to be enhanced by more than 14000 times. The optical impedance spectroscopy with the SM-EA-IOW ${ }^{25}$ demonstrated the capability to detect and follow the electrochemical properties of redox species at very low surface densities, as low as $10^{-15} \mathrm{~mol} / \mathrm{cm}^{2}$.

In this work, we first describe a theoretical formalism that improves accuracy and simplifies the determination of electrontransfer rates from potential modulated data. Next, the SM-EAIOW technology under potential modulation was applied to investigate the electrochemical properties of cytochrome $c$ assemblies at different environmental conditions on the electrode surface and at very low surface densities of redox species, which is a scenario that typically cannot be properly accessed by conventional methodologies due to lack of sensitivity. First, we studied the effects of different bulk volume concentrations of the protein dissolved on a specific buffer solution. Next, we investigated buffer solutions with different ionic strengths while the bulk volume concentration of the protein was kept constant. The impact of those conditions surrounding the working electrode on the electron-transfer rate of the redox assembly is reported in this work. 


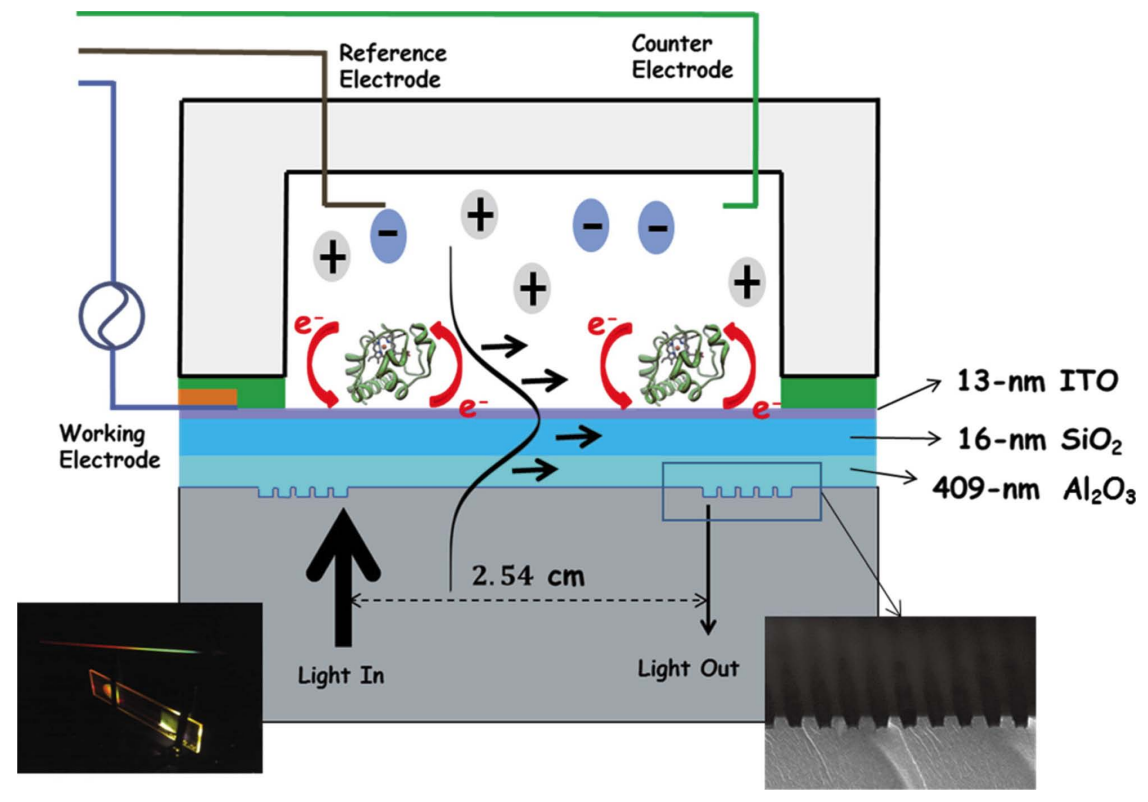

Fig. 1 Schematic representation of the single-mode, electro-active, integrated optical waveguide platform for sensitive spectroelectrochemical measurements of redox events.

\section{Theory}

As mentioned, we have previously ${ }^{25}$ described an overall methodology to obtain electron-transfer rate of redox events from optical absorbance measurements that are driven by an AC modulation of an applied potential. Therefore, here we restrict our theoretical description to a particular simplification of the previously developed formalism.

The electrical response of an electrochemical cell with its working, reference, and counter electrodes, the electrolyte solution, and the redox species can be described by a circuit schematically shown in Fig. 2. Generalized impedances are written as $Z_{1}$ and $Z_{2}$ and they account for the electrolyte solution resistance and other possible electrical effects in the cell. As already shown elsewhere ${ }^{25}$ and confirmed below, specific knowledge of the nature and value of these components are not needed. The applied potential modulation across the whole electrochemical cell, which is provided by the potentiostat, is denoted as $E_{\text {ac }}$ and the associated total electrical impedance, $Z_{\mathrm{t}}$, across the cell is given by $Z_{\mathrm{t}} \equiv \frac{E_{\mathrm{ac}}}{I_{\mathrm{t}}}$, where $I_{\mathrm{t}}$ is the total electric current in the circuit.

Now, as shown in Fig. 2, the potential modulation applied across the redox adsorbate is represented by $E_{\mathrm{ac}, \mathrm{F}}$ and the electric current $I_{\mathrm{F}}$ of the faradaic chemical process can be represented electrically through a charge-transfer resistance, $R_{\mathrm{ct}}$, in series with a pseudo-capacitance, $C_{\mathrm{a}}{ }^{26}$ Those electrical components of the faradaic process then form an admittance $Y_{\mathrm{F}}(\omega) \equiv$ $\frac{I_{\mathrm{F}}}{E_{\mathrm{ac}, \mathrm{F}}}=\left(R_{\mathrm{ct}}+\frac{1}{j \omega C_{\mathrm{a}}}\right)^{-1}$ whose real $Y_{\mathrm{F}}^{\prime}(\omega)$ and imaginary, $Y_{\mathrm{F}}^{\prime \prime}(\omega)$ parts are respectively described by Eqs. (1) and (2):

$$
Y_{\mathrm{F}}^{\prime}(\omega)=\frac{R_{\mathrm{ct}}}{\left(R_{\mathrm{ct}}{ }^{2}+\frac{1}{\omega^{2} C_{\mathrm{a}}^{2}}\right)}
$$

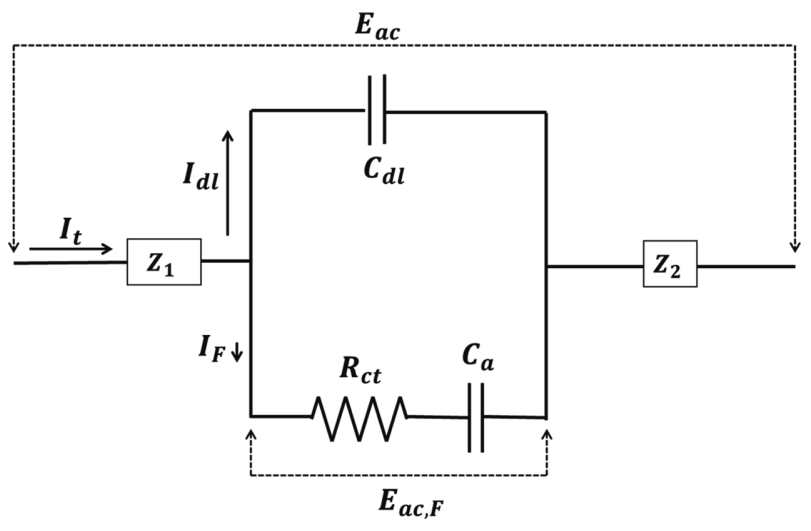

Fig. 2 Electrical circuit for the electrochemical cell in the presence of redox species on the electrode surface. An AC electric potential $E_{\mathrm{ac}}$ is applied across the entire electrochemical cell and creates a total current $I_{\mathrm{t}}$ with an impedance $Z_{\mathrm{t}} \equiv \frac{E_{\mathrm{ac}}}{I_{\mathrm{t}}}$. The potential modulation applied to the redox process is $E_{\mathrm{ac}, \mathrm{F}}$ with a faradaic current $I_{\mathrm{F}}$ and an admittance $Y_{\mathrm{F}} \equiv \frac{I_{\mathrm{F}}}{E_{\mathrm{ac}, \mathrm{F}}}$. A charge-transfer resistance $R_{\mathrm{ct}}$ in series with a pseudo-capacitance $C_{\mathrm{a}}$ account for the electrical admittance $Y_{\mathrm{F}}$ of the faradaic chemical process. In parallel to the redox process, an electric current $I_{\mathrm{dl}}$ goes through the electric double-layer, which is represented by a capacitance $C_{\mathrm{dl}}$. The terms $Z_{1}$ and $Z_{2}$ are generalized impedances, which can account for solution resistance and other effects on the electrochemical cell.

$$
Y_{\mathrm{F}}^{\prime \prime}(\omega)=\frac{1}{\omega C_{\mathrm{a}}\left(R_{\mathrm{ct}}{ }^{2}+\frac{1}{\omega^{2} C_{\mathrm{a}}^{2}}\right)}
$$

where $\omega$ is the angular frequency of the applied potential modulation. Those terms correspond to an in-phase response, $Y_{\mathrm{F}}^{\prime}(\omega)$, and an out-of-phase response, $Y_{\mathrm{F}}^{\prime \prime}(\omega)$, of the faradaic current $I_{\mathrm{F}}$ due to the driving applied potential, $E_{\mathrm{ac}, \mathrm{F}}$. The time response of the faradaic process, $R_{\mathrm{ct}} C_{\mathrm{a}}$, and the electron-transfer 

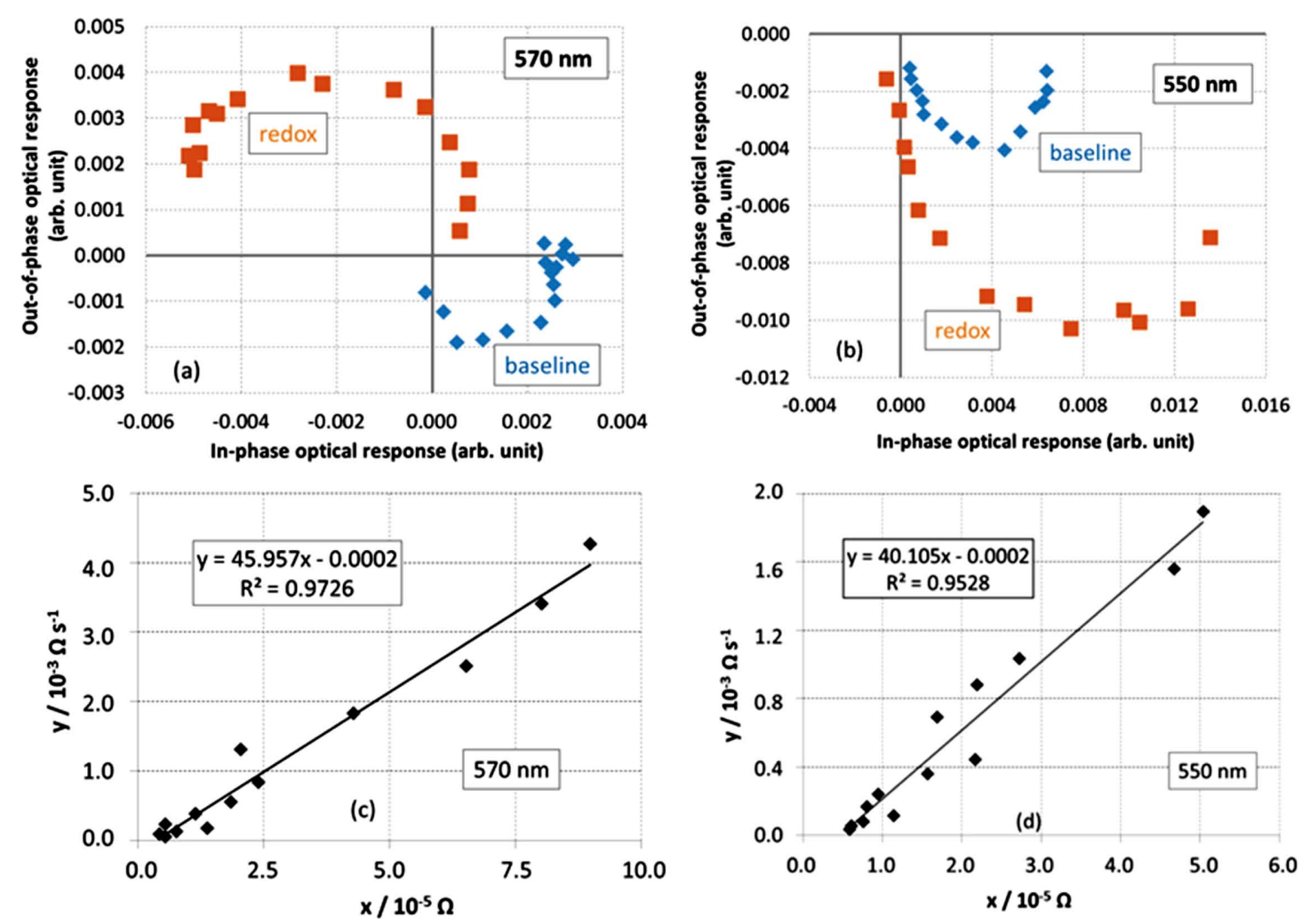

Fig. 3 Optical response in the complex plane for two probing wavelengths: (a) $570 \mathrm{~nm}$ and (b) $550 \mathrm{~nm}$. Blue diamonds: optical response of instrument baseline. Orange squares: optical response when redox species are present in the cell. The modulation frequency increases in the counterclockwise direction. The parametric plot $y=k x$ is shown in (c) for $570 \mathrm{~nm}$ and in (d) for $550 \mathrm{~nm}$ probing wavelength. The slope of a linear fitting to the data displayed in (c) and (d) is used to retrieve electrontransfer rate, $k$.

rate $k \equiv \frac{1}{2 C_{\mathrm{a}} R_{\mathrm{ct}}}$ can then be obtained from Eqs. (1) and (2):

$$
k=\frac{1}{2 C_{\mathrm{a}} R_{\mathrm{ct}}}=\frac{\omega Y_{\mathrm{F}}^{\prime \prime}(\omega)}{2 Y_{\mathrm{F}}^{\prime}(\omega)}
$$

Whenever the faradaic electric current is small compared to the electric double-layer current, the admittance of the faradaic process, $Y_{\mathrm{F}}(\omega)$, was shown ${ }^{25}$ to be described in terms of the faradaic current density, $i_{\mathrm{F}}$, and the total electrical impedance across the cell, $Z_{\mathrm{t}}$, by the following equation:

$$
Y_{\mathrm{F}}(\omega)=j \frac{\omega C_{\mathrm{dl}} A_{\mathrm{eff}}}{E_{\mathrm{ac}}} Z_{\mathrm{t}} i_{\mathrm{F}}
$$

where $A_{\text {eff }}$ is the effective area of the working electrode and $C_{\mathrm{d}}$ is the capacitance of the electric double layer (in parallel with the redox process, see Fig. 2). The faradaic current density $i_{\mathrm{F}}$ is measured through the modulated optical absorbance signal by $i_{\mathrm{F}}=\frac{n F}{S \Delta \varepsilon} \frac{\mathrm{d} A}{\mathrm{~d} t}=\frac{n F}{S \Delta \varepsilon} j \omega A_{\mathrm{ac}}$, where $S$ is a sensitivity factor (e.g., $S \cong 14500$ for the SM-EA-IOW device), $\Delta \varepsilon$ is the difference in molar absorptivity between the two oxidation states, $n$ is the number of electrons involved in the redox process, and $F$ is the Faraday constant. By inserting this result into Eq. (4), we get:

$$
\begin{array}{r}
Y_{\mathrm{F}}(\omega)=-\frac{n F \omega^{2} C_{\mathrm{dl}} A_{\mathrm{eff}}}{S \Delta \varepsilon \Delta E_{\mathrm{ac}}} \times\left\{\left[Z_{\mathrm{t}}^{\prime}(\omega) A_{\mathrm{ac}}^{\prime}(\omega)-Z_{\mathrm{t}}^{\prime \prime}(\omega) A_{\mathrm{ac}}^{\prime \prime}(\omega)\right]+\right. \\
\left.j\left[Z_{\mathrm{t}}^{\prime}(\omega) A_{\mathrm{ac}}^{\prime \prime}(\omega)+Z_{\mathrm{t}}^{\prime \prime}(\omega) A_{\mathrm{ac}}^{\prime}(\omega)\right]\right\}
\end{array}
$$

By defining

$$
y(\omega) \equiv \omega\left[Z_{\mathrm{t}}^{\prime}(\omega) A_{\mathrm{ac}}^{\prime \prime}(\omega)+Z_{\mathrm{t}}^{\prime \prime}(\omega) A_{\mathrm{ac}}^{\prime}(\omega)\right]
$$

and

$$
x(\omega) \equiv 2\left[Z_{\mathrm{t}}^{\prime}(\omega) A_{\mathrm{ac}}^{\prime}(\omega)-Z_{\mathrm{t}}^{\prime \prime}(\omega) A_{\mathrm{ac}}^{\prime \prime}(\omega)\right]
$$

then Eq. (3) gives us $y(\omega)=k x(\omega)$. Thus, in a parametric plot of $y(\omega)$ against $x(\omega)$, the slope of the linear fit will provide the electron-transfer rate, $k$, of the faradaic process. Although the faradaic impedance, $Y_{\mathrm{F}}(\omega)$, depends on the values of $C_{\mathrm{dl}}, A_{\mathrm{eff}}, \Delta \varepsilon$ and other constants, those quantities are not directly involved in the determination of the rate constant, $k$. Therefore, for the purpose of determining the electron-transfer rate, $k$, the analysis above shows that it is sufficient to collect the electrical impedance of the electrochemical cell, $Z_{\mathrm{t}}(\omega)$, and the modulated absorbance signal, $A_{\mathrm{ac}}(\omega)$. The electrical impedance across the electrochemical cell, $Z_{\mathrm{t}}(\omega)$, is usually obtained via a potentiostat driving the potential applied to the cell and measuring the total output current, $I_{\mathrm{t}}$. The modulated absorbance, $A_{\mathrm{ac}}(\omega)$, is obtained by measuring a probing optical signal with a photo-multiplier connected to a lock-in-amplifier that has been synchronized to the driving potential of the potentiostat.

\section{Experimental Results}

To exemplify the benefits of the above methodology, we have 

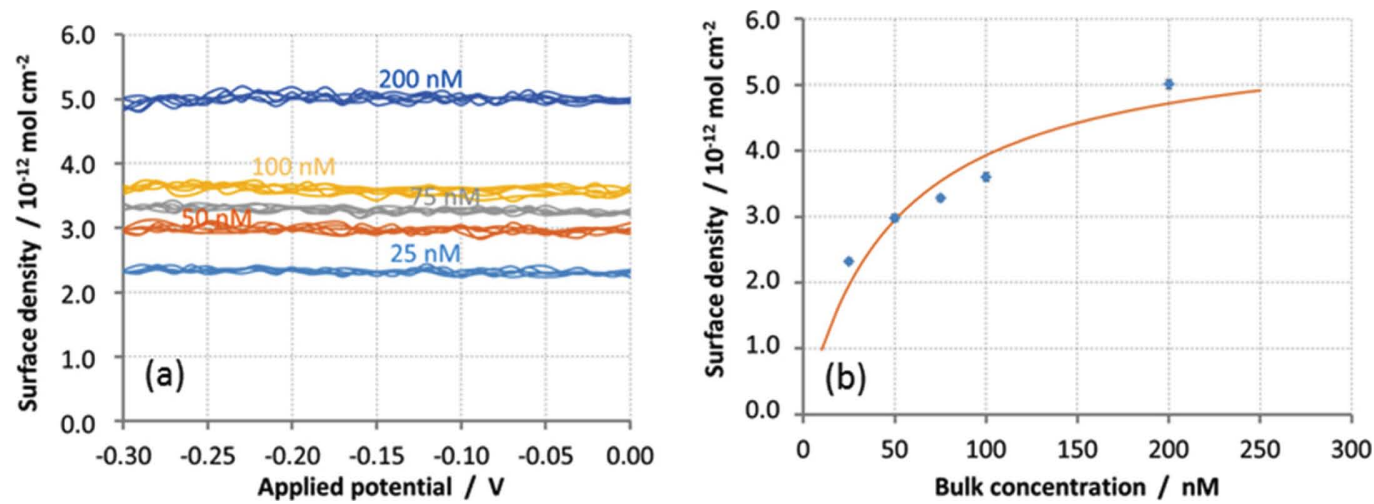

Fig. 4 (a) Surface density of cytochrome $c$ during cyclic voltammetry scans is plotted for each cytochrome $c$ bulk concentration at an isosbestic wavelength. (b) Surface density of the redox molecules against bulk concentration and a Langmuir fit to the experimental data.

chosen two probing wavelengths that our model redox molecule (cytochrome $c$ protein) show a very distinct optical behavior. During a transition from an oxidized to a reduced state this redox probe shows a negative change in molar absorptivity $\left(\Delta \varepsilon=\varepsilon_{\text {red }}-\varepsilon_{\text {ox }}=-4 \times 10^{3} \mathrm{M}^{-1} \mathrm{~cm}^{-1}\right)$ at $570 \mathrm{~nm}$ while at $550 \mathrm{~nm}$ it shows a positive change $\left(\Delta \varepsilon=\varepsilon_{\text {red }}-\varepsilon_{\text {ox }}=18 \times 10^{3} \mathrm{M}^{-1} \mathrm{~cm}^{-1}\right)$. The optical signals measured by the SM-EA-IOW platform (see Fig. 1) are displayed (in the complex plane) in Figs. 3(a) and 3(b) for the 570 and $550 \mathrm{~nm}$, respectively, and each data point corresponds to a particular modulation frequency, $\omega$, of the applied potential. The optical response of the baseline signal collected with the SM-EA-IOW platform at 570 and $550 \mathrm{~nm}$, which are represented by blue diamonds in Figs. 3(a) and 3(b), shows a similar pattern mostly located in the fourth quadrant. On the other hand, the optical signal in the presence of redox species, which are represented by orange squares in those figures, shows a remarkable difference between these two wavelengths. Despite such clear differences, when the formalism described above for the determination of the electrontransfer rate, $k$, was applied, consistent results were obtained as summarized in Fig. 3(c) for $570 \mathrm{~nm}$ with $k=(46 \pm 2) s^{-1}$ and in Fig. 3(d) for $550 \mathrm{~nm}$ with $k=(40 \pm 3) s^{-1}$. These experimental results illustrate the consistency of the simplified methodology for determination of the electron-transfer rate of the redox process.

Next we apply the SM-EA-IOW technology to investigate the impact on the electron-transfer rate of different conditions surrounding the interaction of redox species with the indium tin oxide (ITO) electrode of a SM-EA-IOW device. Our model redox molecule is the cytochrome $c$ protein dissolved in phosphate buffered saline (PBS) solution. Detailed description of our experimental conditions can be found in a previous report. ${ }^{25}$

Electron-transfer rate at different surface densities of redox species on the electrode

Different surface densities of redox species on the electrode were achieved by using PBS solutions with different concentrations of the cytochrome $c$ protein. To determine the density of surface-bound molecules, a cyclic voltammetry (CV) modulation with a scanning range from -0.3 to $0.0 \mathrm{~V}$, a scan rate of $20 \mathrm{mV} / \mathrm{s}$, and for a total of 3 cycles were used while broadband light was propagating along the SM-EM-IOW and the outcoupled optical signal was spectrally resolved by a CCD camera. The isosbestic wavelength at $556.5 \mathrm{~nm}$, which largely simplifies the calculations, was selected to determine the surface density from the measured optical absorbance data and to monitor any possible net adsorption or desorption from the electrode surface of cytochrome $c$ molecules during potential modulation. To determine the electron-transfer rate, $k$, an AC impedance potential modulation $(15 \mathrm{mV}$ amplitude) with a single probing wavelength $(550 \mathrm{~nm})$ was deployed.

Initially, optical baselines for $\mathrm{CV}$ and $\mathrm{AC}$ experiments were collected after a PBS solution $(\mathrm{pH} \cong 7.2$, concentration $=$ $50 \mathrm{mM}$ ) was injected into the electrochemical flowcell. Then different concentrations of cytochrome $c$ protein $(25,50,75$, 100 and $200 \mathrm{nM}$ ) were sequentially tested. First, an amount of $2 \mathrm{~mL}$ of cytochrome $c$ solution at $25 \mathrm{nM}$ was injected into the electrochemical flowcell and let it incubate for a $30-\mathrm{min}$ period. Then, a rinsing procedure with PBS solution was used to eliminate unbound cytochrome $c$ molecules. Next, the same potential modulations ( $\mathrm{CV}$ and $\mathrm{AC}$ ) were applied to collect data in the presence of the analyte. Without disassembling the flowcell but after rinsing it with PBS solution, each solution with the next higher protein concentration was sequentially injected, incubated, and data under $\mathrm{CV}$ and $\mathrm{AC}$ potential modulation were collected.

From the optical data at CV modulation, the surface density at each volume concentration of the redox solution is plotted against the applied potential and shown in Fig. 4(a). As observed from these experimental results, the surface density for each bulk concentration can be considered almost constant during the $\mathrm{CV}$ potential scans. Therefore, there is no net adsorption or desorption of redox species from the electrode surface during potential modulation. In Fig. 4(b), the surface density $\Gamma$ data is plotted against the bulk concentration $C_{\mathrm{b}}$ of the redox solution in combination with a Langmuir fit, ${ }^{27}$ as described by Eq. (8). From the fitting results, we have determined a surface density saturation of $\Gamma_{\text {sat }}=(5.9 \pm 0.7) \mathrm{pmol} / \mathrm{cm}^{2}$ and an adsorption constant of $K_{\mathrm{ad}}=(2.3 \pm 0.8) \times 10^{7} \mathrm{M}^{-1}$.

$$
\Gamma=\frac{K_{\mathrm{ad}} \Gamma_{\mathrm{sat}} C_{\mathrm{b}}}{1+K_{\mathrm{ad}} C_{\mathrm{b}}}
$$

From the AC impedance data and the formalism described in the Theory section the electron-transfer rate under different densities of redox molecules on the electrode surface was determined; these experimental results are summarized in Fig. 5(a). At low surface densities the electron-transfer rate, $k$, can be considered as approximately constant but its value is consistently reduced as the surface density increases. 

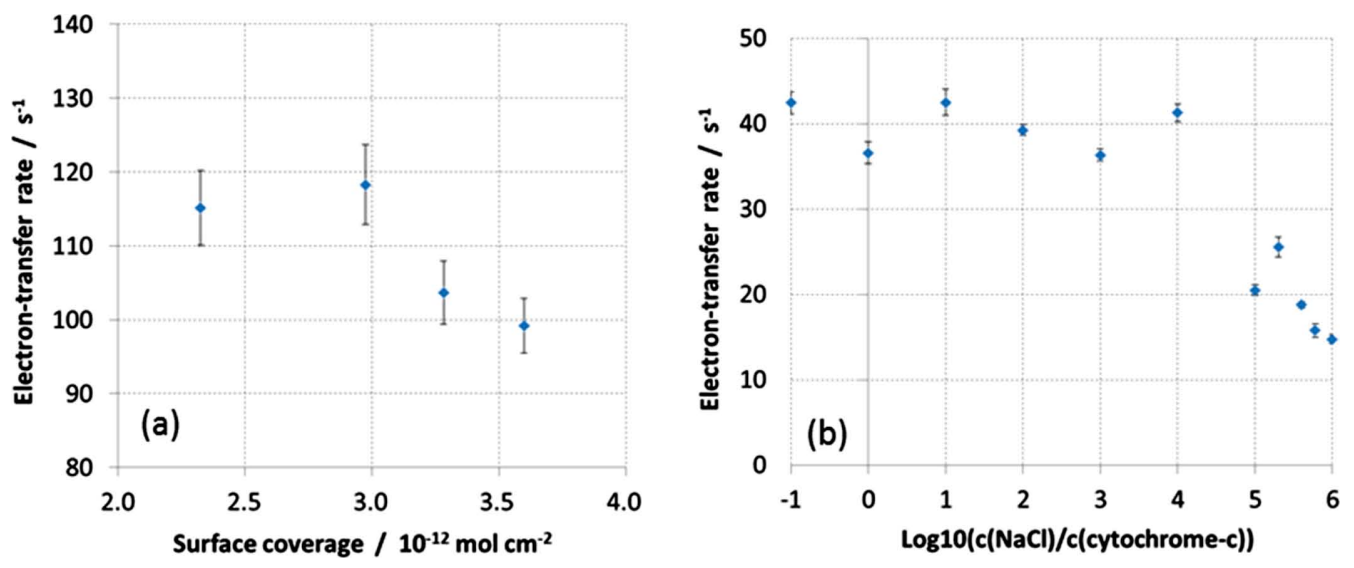

Fig. 5 (a) Electron-transfer rate of surface-immobilized cytochrome $c$ molecules under different surface densities. (b) Electron-transfer rate of cytochrome $c$ molecules from solutions with different ionic strengths.

\section{Electron-transfer rate with electrolyte solutions of different ionic strength}

The ionic strength was adjusted through the concentration of the $\mathrm{NaCl}$ salt in the PBS solution. The bulk concentration of cytochrome $c$ protein was fixed at $100 \mathrm{nM}$. For each salt concentration, the optical baseline was taken separately. After a cleaning procedure, the PBS solution at a specific concentration of $\mathrm{NaCl}$ was injected into the flowcell. An $\mathrm{AC}$ impedance potential modulation was applied to record the optical baseline signal with a probing wavelength at $570 \mathrm{~nm}$. Next, a protein solution under the same salt concentration was injected and let it to incubate for a $30-\mathrm{min}$ period. After the immobilization, a similar AC impedance potential modulation was applied to the SM-EA-IOW device and data was recorded. In Fig. 5(b), the electron-transfer rate is plotted against the logarithm of the ratio of the concentration of $\mathrm{NaCl}$ over the concentration of cytochrome $c$ protein. For the case of no salt present in the PBS solution, we used the $x$-coordinate at -1 (instead of $-\infty$ ) to represent this particular data point. When the $\mathrm{NaCl}$ concentration is low (from 0 to $1 \mathrm{mM}$ ), the electron-transfer rate is almost a constant. When $\mathrm{NaCl}$ concentration is increased above the $\mathrm{mM}$ range, the electron-transfer rate is reduced almost monotonically. It is worth mentioning that in the case when the $\mathrm{NaCl}$ concentration in the PBS solution was higher than $100 \mathrm{mM}$, we observed that the cytochrome $c$ molecules could not be immobilized on the ITO electrode surface.

\section{Discussions}

Our data show that both the surface density of redox molecules and the ionic strength of the electrolyte solution have a clear influence on the rate of electron transfer between the ITO electrode and the cytochrome $c$ redox molecules. At low surface densities or low ionic strength, a higher value of the electrontransfer rate was determined. It is known that at our almost neutral $\mathrm{pH}$ conditions the main binding mechanism between the ITO electrode and the cytochrome $c$ molecules is through the electrostatic force. The experimental results described above can be attributed to the binding strength featured by different sites present on the surface of the ITO electrode. The ITO surface cannot be considered a homogeneous interface. In simple terms, one should consider sites that offer strong binding energy while other sites may just provide a weak binding affinity. The difference in binding strength across the ITO surface can come from a variation in the stoichiometry and in the degree of oxidation across the film with an impact on the two-dimensional charge distribution over the electrode surface. At some local regions, the density of negative charges can be higher and generate a strong binding site for the positively charged cytochrome $c$ protein. At other spots, the density of negative charges can be lower and generate just a weak binding site. Although working with a different interface, the impact of the surface heterogeneity in the electron-transfer rate of redox proteins has been observed by others. ${ }^{28}$

For the experiment with different concentrations of the redox species, one can consider that at low surface densities the molecules will bind primarily to the strong binding sites as they are abundantly available. And a strong binding interaction between the ITO electrode and the redox molecules is expected to provide a faster electron-transfer process. When the volume (and surface) concentration of redox molecules is increased, a fraction of the surface-adsorbed species will start occupying sites that feature weaker (less strong) interaction energy. On average the binding strength between the electrode and the surface-bound molecules will decrease and lead to a reduction in electron-transfer rate. An illustration of the effect of the binding strength on the electron-transfer rate is displayed in Fig. 6(a) at low surface density and in Fig. 6(b) at high surface density cases.

For the experiment with different ionic strengths of the electrolyte solution, sodium and chlorine ions can affect both the cytochrome $c$ molecules and the binding sites of the ITO electrode. As the salt concentration is increased, positively charged $\mathrm{Na}$ cations in the electrolyte solution will compete for the binding sites on the electrode surface and will tend to neutralize its surface charge. In addition, the negatively charged $\mathrm{Cl}$ anions will form a shielding shell on the cytochrome $c$ molecules that will reduce its binding strength to the electrode. In this case, redox species can only loosely attach to the surface of the device and the electron-transfer rate is expected to decrease. If the $\mathrm{NaCl}$ concentration is excessively high, cytochrome $c$ proteins can no longer adsorb to the electrode surface. The illustration of this rationale is shown in Fig. 6(c) for low ionic strength and in Fig. 6(d) for high ionic strength.

By comparing the two sets of experiments under the same condition, which corresponds to $100 \mathrm{nM}$ bulk concentration of cytochrome $c$ protein in PBS solution without any additional 
(a)

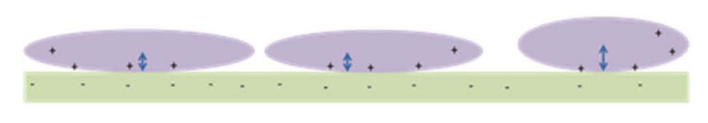

(c)

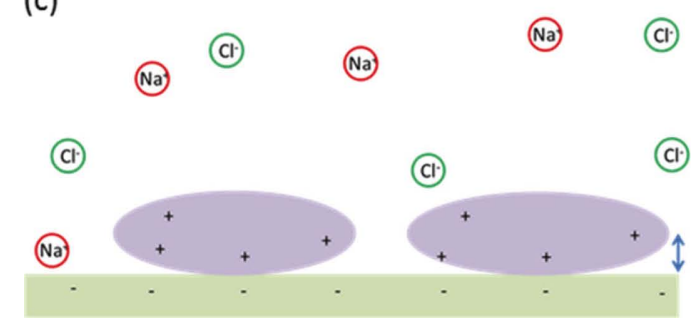

(b)

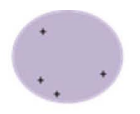

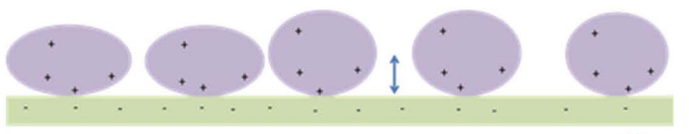

(Na)

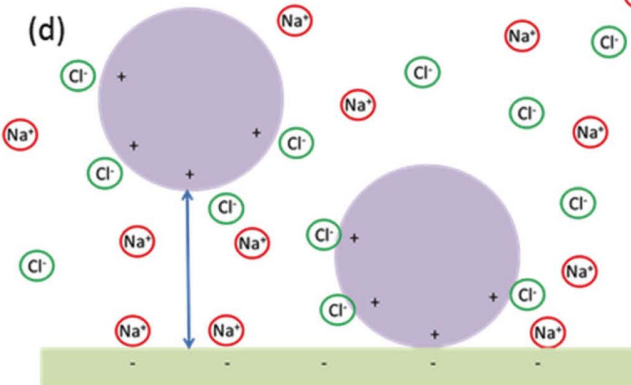

Fig. 6 Illustrations of the impact on the electron-transfer rate from (a) low surface density, (b) high surface density, (c) low ionic strength, and (d) high ionic strength.

$\mathrm{NaCl}$ salt, the effect of different electron-transfer rate can also be observed. Under those conditions, the electron-transfer rate is $99 \mathrm{~s}^{-1}$ for the first set of experiments and $42 \mathrm{~s}^{-1}$ for the second set of experiments. Different SM-EA-IOW devices were used for these two sets of experiments. This result is an additional evidence that the chemistry of the analytical interface is quite important to the electron-transfer process between working electrodes and redox adsorbates.

\section{Conclusions}

In this work we have described a novel methodology to determine electron-transfer rate of redox adsorbates using a combination of impedance data from optical and electrical measurements. The approach is based on the widely accepted assumption of a charge-transfer resistance and a pseudocapacitance to describe in electrical terms an electrochemicaly driven redox process. And it relies on simultaneous acquisition of the electrical impedance across an electrochemical cell, which is routinely performed by a potentiostat, and the modulated optical signal associated with spectroscopic changes of redox species undergoing potential modulation, which can be obtained by a photo-detector connected to a lock-in-amplifier.

The efficiency of the developed methodology was illustrated by collecting optical data with a pair of laser wavelengths that give very distinct optical absorption for a particular redox reaction, and indeed the new formalism still provides good agreement in the determination of the electron-transfer rate. Next, our methodology was put to practice for investigating electron-transfer rate of cytochrome $c$ adsorbates at very low volume and surface concentrations, which is a scenario that largely benefits from the ultra-high sensitivity of the SM-EAIOW platform. Different surface densities of the redox species on the electrode interface and different ionic strengths in the electrolyte solution were investigated. Higher surface densities and higher ionic strengths are shown to slow down on average the electron-transfer process between the redox molecules and the working electrode.

It did not escape our attention that the formalism developed here for determination of electron-transfer rate is fairly general and applicable to any experimental technique where the electrical modulation that drives the oxidation state of the redox species will also stimulate on them a detectable and modulated optical signal. Thus, different optical techniques such as absorbance (in a variety of formats including transmission, reflection, ATR, etc.), fluorescence, and Raman scattering can possibly benefit from the simplicity of the methodology described here to determine time constants of electrically driven redox reactions.

\section{Acknowledgements}

The authors acknowledge financial support from National Institute of Health (NIH Award RR022864), National Science Foundation (NSF EPSCoR Award 0814194), and the Kentucky Science and Engineering Foundation (KSEF Award 1869-RDE012).

\section{References}

1. D. Alvarez-Paggi, W. Meister, U. Kuhlmann, I. Weidinger, K. Tenger, L. Zimanyi, G. Rakhely, P. Hildebrandt, and D. H. Murgida, J. Phys. Chem. B, 2013, 117, 6061.

2. D. A. Capdevila, W. A. Marmisolle, F. J. Williams, and D. H. Murgida, Phys. Chem. Chem. Phys., 2013, 15, 5386.

3. Y. Qian, X. Xu, Q. Wang, P. Wu, H. Zhang, and C. Cai, Phys. Chem. Chem. Phys., 2013, 15, 16941.

4. A. Vertova, A. Forlini, and S. Rondinini, J. Electrochem. Soc., 2012, 159, F81.

5. R. Petrucci, G. Marrosu, P. Astolfi, G. Lupidi, and L. Greci, Electrochim. Acta, 2012, 60, 230.

6. J. Huang, L. Zhang, R. P. Liang, and J. D. Qiu, Biosens. Bioelectron., 2013, 41, 430.

7. M. K. Beissenhirtz, F. W. Scheller, and F. Lisdat, Anal. Chem., 2004, 76, 4665.

8. T. Kuwana and N. Winograd, in "Electroanalytical Chemistry", ed. A. Bard, 1974, Dekker, New York.

9. W. R. Heineman, F. M. Hawkridge, and H. N. Blount, in "Electroanalytical Chemistry", ed. A. Bard, 1984, Dekker, 
New York.

10. Z. Q. Feng, T. Sagara, and K. Niki, Anal. Chem., 1995, 67, 3564.

11. T. Sagara, S. Igarashi, H. Sato, and K. Niki, Langmuir, 1991, 7, 1005.

12. T. Amemiya, K. Hashimoto, and A. Fujishima, Denki Kagaku, 1992, 60, 1075.

13. T. Amemiya, K. Hashimoto, and A. Fujishima, J. Phys. Chem., 1993, 97, 9736.

14. T. Amemiya, K. Hashimoto, and A. Fujishima, $J$. Electroanal. Chem., 1994, 377, 143.

15. T. Amemiya, K. Hashimoto, A. Fujishima, and K. Itoh, $J$. Electrochem. Soc., 1991, 138, 2845.

16. T. Ruzgas, L. Wong, A. K. Gaigalas, and V. L. Vilker, Langmuir, 1998, 14, 7298.

17. L. Li, C. Meuse, V. Silin, A. K. Gaigalas, and Y. Z. Zhang, Langmuir, 2000, 16, 4672.

18. Z. O. Araci, A. F. Runge, W. J. Doherty, 3rd, and S. S. Saavedra, J. Am. Chem. Soc., 2008, 130, 1572.

19. Z. O. Araci, A. F. Runge, W. J. Doherty, and S. S. Saavedra
Israel Journal of Chemistry, 2006, 46, 249.

20. Z. O. Araci, C. R. Shallcross, N. R. Armstrong, and S. S. Saavedra, J. Phys. Chem. Lett., 2010, 1, 1900.

21. D. R. Dunphy, S. B. Mendes, S. S. Saavedra, and N. R. Armstrong, Anal. Chem., 1997, 69, 3086.

22. J. T. Bradshaw, S. B. Mendes, N. R. Armstrong, and S. S. Saavedra, Anal. Chem., 2003, 75, 1080.

23. M. M. Aslan, N. A. Webster, C. L. Byard, M. B. Pereira, C. M. Hayes, R. S. Wiederkehr, and S. B. Mendes, Thin Solid Films, 2010, 518, 4935.

24. X. Han and S. B. Mendes, "Studies of Redox Reaction in Electro-active Proteins Using Optical Impedance Spectroscopy at Single-Mode Waveguides", in SPIE, 2013.

25. X. Han and S. B. Mendes, Anal. Chem., 2014, 86, 1468.

26. T. Sagara, K. Niwa, A. Sone, C. Hinnen, and K. Niki, Langmuir, 1990, 6, 254

27. A. W. Adamson and A. P. Gast, "Physical Chemistry of Surfaces", 6th ed., 1997, Wiley-Interscience.

28. R. A. Clark and E. F. Bowden, Langmuir, 1997, 13, 7. 